\title{
Produtividade total dos fatores e acumulação de capital no Brasil
}

Roberto Ellery Jr*

\begin{abstract}
Resumo: Este trabalho tem como objetivo avaliar o papel da produtividade total dos fatores (PTF) na determinação do produto e do investimento no Brasil. Parte significativa da discussão sobre o papel da PTF na determinação do produto é feita por meio de exercícios de contabilidade do crescimento, apesar de oferecer informações importantes esta técnica não permite avaliar o quanto do ganho de produtividade pode ser advindo do acumulo de capital e o quanto do aumento no estoque de capital pode ser devido ao aumento da produtividade. Para avaliar o papel da produtividade na determinação do capital são feitas simulações do modelo básico de crescimento, calibrado para reproduzir a economia brasileira, porém com diferentes hipóteses comportamento para a evolução da PTF. Resultados preliminares mostram que parte da acumulação de capital pode ser explicada pela PTF, desta forma políticas que visem aumentar a produtividade podem, além do efeito direto sobre o crescimento, elevar o investimento causando um impacto final maior do que o suposto nos exercícios de contabilidade do crescimento.
\end{abstract}

\begin{abstract}
This study aims to evaluate the role of total factor productivity (TFP) in the determination of output and investment in Brazil. A significant part of the discussion on the role of TFP in the determination of the product is made by means of growth accounting exercises, while offering important information this technique does not allow to assess the extent in witch productivity gains may be coming from the accumulation. To evaluate the role of productivity in determining the capital accumulation I run simulations of the basic model of growth, calibrated to reproduce the Brazilian economy, but with different hypotheses for the evolution behavior of TFP. Preliminary results show that part of capital accumulation can be explained by TFP, so policies to increase productivity may, in addition to the direct effect on growth, increase investment.
\end{abstract}

Palavras-chave: Investimento; Produtividade; Brasil.

Classificação JEL: D91; D92; C61.

"Doutor em Economia pela Universidade de Brasília (UNB). Professor adjunto do Departamento de Economia da Universidade de Brasília (UNB). Endereço eletrônico: ellery@unb.br. 


\section{Introdução}

Um tema central na discussão sobre o desempenho econômico do Brasil e da América Latina diz respeito aos papéis da acumulação de capital e da produtividade total dos fatores (PTF) na trajetória de longo prazo do produto per-capita. A literatura sugere que o crescimento brasileiro, em sua maior parte, é devido à acumulação de capital, esta característica é comum a outros países da América Latina, porém contrasta com o padrão de crescimento do Leste Asiático'.

Um ponto relevante é a relação entre produtividade e investimento. Naturalmente, estas duas variáveis possuem relativa interdependência, de forma que nem sempre é possível separar o papel de cada uma do processo de desenvolvimento econômico. A idéia de que muitas vezes os ganhos de produtividade só podem ser obtidos por meio de investimentos, como no caso de tecnologias embutidas em novas gerações de máquinas, é relativamente bem explorada na literatura, entretanto, menos esforço foi feito no sentido de avaliar o impacto dos ganhos de produtividade sobre a decisão de investir.

Este trabalho procura avaliar como o comportamento da PTF influenciou o investimento no Brasil. Seguindo a metodologia utilizada em Kehoe e Prescott (2007) serão feitas simulações do modelo neoclássico de crescimento ${ }^{2}$ tomando como exógeno o comportamento da PTF. Desta forma será possível avaliar como mudanças na produtividade poderiam ter afetado o produto e a taxa de investimento no Brasil. Neste sentido o trabalho segue a linha desenvolvida em Bugarin et alli (2007) e Ellery Jr e Teixeira (2013) e avança no sentido de focar nos efeitos da PTF sobre o investimento no Brasil.

$\mathrm{Na}$ próxima seção serão apresentados exercícios de contabilidade do crescimento. Na terceira seção será apresentado o modelo básico e a relação teórica entre PTF e acumulação de capital. A quarta seção apresenta as simulações do modelo e a quinta seção concluí o trabalho.

\section{Contabilidade do Crescimento}

Suponha que o produto da economia brasileira possa ser descrito por uma função de produção do tipo Cobb-Douglas ${ }^{3}$ do tipo:

$$
Y_{t}=A_{t} K_{t}^{\theta} L_{t}^{1-\theta}
$$

Onde $Y_{t}$ representa o produto agregado, $A_{t}$ a produtividade total dos fatores (PTF), $K_{t}$ o estoque de capital, $L_{t}$ a quantidade de trabalho utilizada na produção e $\theta$ é um parâmetro tecnológico que determina a participação da renda do capital na renda total. Conhecidos os valores de $Y_{t}, K_{t}, L_{t}$ e do parâmetro $\theta$ é possível de terminar a produtividade total dos fatores.

A série de produto utilizada foi a do Produto Interno Bruto das Contas

1 A este respeito ver Gomes, Pessoa e Veloso (2003), para uma visão alternativa sobre o papel da produtividade no leste da Ásia ver Young (1995).

2 Ver Cooley e Prescott (1995).

3 Sobre a adequação do uso de funções de Cobb-Douglas ver Pessoa et al (2003), para o caso do Brasil ver Ellery Jr et al (2002). 
Nacionais, para o estoque de capital utilizou-se a série do IPEADATA descrita em Morandi e Reis (2004) e atualizada até 2005, para o insumo trabalho foi utilizado o pessoal ocupado de acordo com as Contas Nacionais ${ }^{4}$. O valor da participação da renda do capital na renda total, $\theta$, segue Gomes et al (2003 e 2005) e foi fixado em 0,40 , número próximo ao sugeridos pela evidência internacional ${ }^{5}$.

O Gráfico 1 apresenta a evolução da PTF, da relação capital trabalho e do produto per-capita no Brasil entre 1950 e 2005. Note-se que enquanto a PTF cresce aproximadamente $48 \%$ em todo o período o produto per-capita cresce de $310 \%$ e a relação capital trabalho cresce mais de $450 \%$. A Figura 1 ilustra a estagnação relativa da PTF em relação ao produto per-capita no Brasil e deixa claro o pequeno papel que a produtividade teve no crescimento da economia brasileira no pós-guerra.

Gráfico 1 - PTF, Relação Capital Trabalho e Produto per-capita, Brasil 1950 - 2005

$$
1950=100
$$

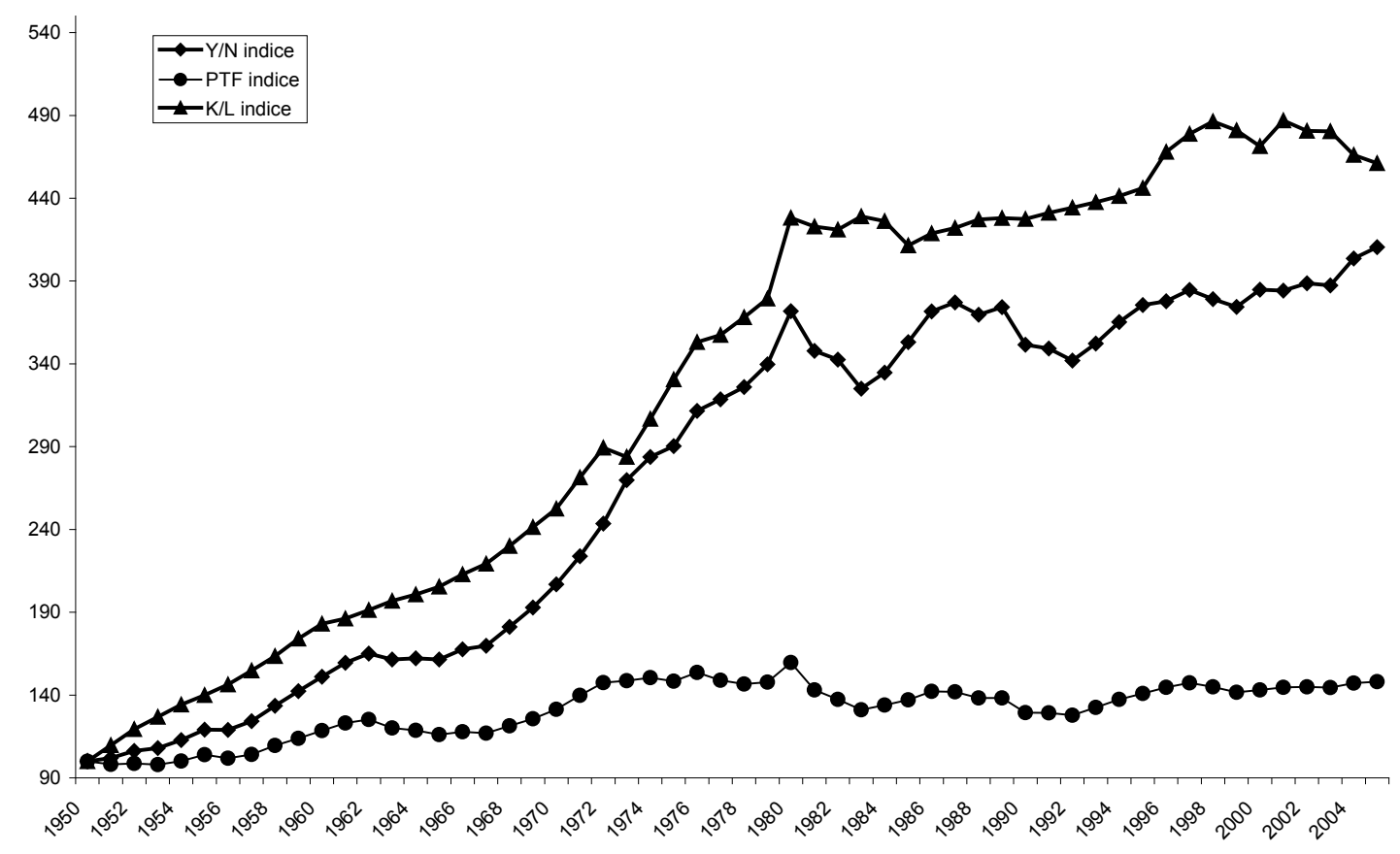

Partindo de 100 em 1950 o valor da PTF apresenta trajetória crescente até 1974, quando chega ao valor de 150,5; a partir deste ponto apresenta trajetória irregular com um pico em 1980 de 159,7, maior valor de toda a série. A década de 1980 foi de queda na PTF com uma lenta recuperação a partir de meados dos anos 1990, de forma que apenas em 2005 retorna aos valores de 1974.

Em claro contraste com este comportamento aparece a relação capital

\footnotetext{
4 Foram as usadas as Contas Nacionais referência 1985 para construir a série até 2003, os anos de 2004 e 2005 só estão disponíveis com as Contas Nacionais referência 2002. Como forma de evitar descontinuidade a taxa de crescimento do pessoal ocupado entre 2003 e 2004 e 2004 e 2005 foi utilizada para completar a série até 2005. 5 Ver Gollin (2002).
} 
trabalho, esta apresenta crescimento praticamente ininterrupto até 1980, sofrendo pequeno efeito da crise do petróleo no início dos anos 1970, e já em 1988 havia retornado ao nível de 1980. O produto tem um comportamento semelhante ao da relação capital trabalho, já em meados dos anos 1986 havia retornado aos níveis de 1980, seguindo trajetória irregular até 1994 e chegando ao seu pico em 2005.

A análise da Figura 1, embora limitada, aponta na direção clara de que o crescimento da economia brasileira está mais relacionado à acumulação de capital do que ao incremento da produtividade. Visando aprofundar a discussão, visto que análises gráficas podem ser enganadoras, será feito um exercício de contabilidade do crescimento para a economia brasileira no período em questão.

Para realizar o exercício de contabilidade do crescimento o produto percapita será representado na forma:

$$
\frac{Y_{t}}{N_{t}}=A_{t}\left(\frac{K_{t}}{L_{t}}\right)^{\theta}\left(\frac{L_{t}}{N_{t}}\right)
$$

Onde $N_{t}$ representa a população residente.

Tomando-se logaritmo da equação 2 e calculando as taxas de crescimento é possível decompor a taxa de crescimento do produto per-capita entre as taxas de crescimento da PTF, da relação capital trabalho e do trabalho. A equação 3 representa esta decomposição:

$$
\gamma_{\mathrm{N}} \approx \gamma_{A}+\theta \gamma_{K}+\gamma_{N}
$$

Onde $\gamma_{\mathbb{N}}$ representa a taxa de crescimento do produto per-capita, $\gamma_{A}$ a taxa de crescimento da PTF, $\gamma_{\mathbb{K}}$ a taxa de crescimento da relação capital trabalho e $\gamma_{N}$ a taxa de crescimento do esforço de trabalho.

A Tabela 1 apresenta a contabilidade do crescimento para o Brasil entre 1950 e 2005 . Até a década de 1990 a contribuição dos fatores de produção supera a contribuição da PTF ${ }^{6}$. Entre 1950 e 1970 a acumulação relativa de capital foi a maior responsável pelo crescimento da economia brasileira. A partir da década de 1970 o crescimento da força de trabalho torna-se o maior responsável pelo crescimento, este fato captura o papel das grandes migrações que ocorreram no período. Note-se que o crescimento da força de trabalho foi também o responsável pelo crescimento positivo na década de 1980.

6 Este resultado é comum a outras analises semelhantes realizadas para o Brasil e para a América Latina, a este respeito ver Gomes et al (2003). Para uma análise crítica a exercícios de contabilidade do crescimento nos moldes realizados nesta seção ver Young (1995). 


\begin{tabular}{ccccc} 
Tabela 1 - Contabilidade de Crescimento, Brasil - $1950-2005$ \\
\cline { 3 - 5 } & Taxa de Cresc. & \multicolumn{3}{c}{ Devido a: } \\
\cline { 3 - 5 } PIBpc & PTF & K/L & L/N \\
\hline $1950-59$ & $4,00 \%$ & $1,45 \%$ & $2,54 \%$ & $0,02 \%$ \\
$1960-69$ & $2,74 \%$ & $0,66 \%$ & $1,25 \%$ & $0,82 \%$ \\
$1970-79$ & $5,66 \%$ & $1,31 \%$ & $1,85 \%$ & $2,43 \%$ \\
$1980-89$ & $0,07 \%$ & $-1,59 \%$ & $0,00 \%$ & $1,69 \%$ \\
$1990-99$ & $0,70 \%$ & $1,01 \%$ & $0,53 \%$ & $-0,83 \%$ \\
$2000-05$ & $1,30 \%$ & $0,69 \%$ & $-0,18 \%$ & $0,78 \%$ \\
$1950-05$ & $2,60 \%$ & $0,72 \%$ & $1,13 \%$ & $0,74 \%$ \\
\hline
\end{tabular}

Fonte: Elaboração própria como base nos dados do Ipeadata e

Contas Nacionais.

Os resultados da contabilidade do crescimento corroboram as conclusões da Figura 1. De acordo com Solow (1956 e 1957) no longo prazo a taxa de crescimento da economia é determinada pela taxa de crescimento da produtividade total dos fatores. Neste sentido, o declínio da taxa de crescimento da economia brasileira a partir da década de 1980 é consistente com o proposto em Solow. A estagnação dos anos 1980 foi decorrência da incapacidade da economia brasileira de gerar ganhos de produtividade.

A despeito da previsão teórica, parte significativa das políticas públicas visando crescimento, considera que o aumento da taxa de investimento, ou da taxa de poupança, é o principal objetivo a ser alcançado para que o país volte a crescer. Políticas direcionadas á retomada da produtividade muitas vezes ocupam espaço secundário na discussão sobre crescimento ${ }^{7}$. Um dos possíveis motivos é a falta de consenso teórico a respeito de quais variáveis determinam o desempenho da produtividade, enquanto a taxa de investimento pode ser diretamente afetada por ações do governo.

Entretanto, além de afetar diretamente o crescimento, a PTF afeta a relação capital trabalho. Deste modo, o aumento da produtividade pode elevar a relação capital trabalho, com a vantagem que este aumento seria advindo de decisões ótimas dos agentes econômicos e não de planos de investimento públicos com potencial de distorcer as decisões ótimas dos agentes. A próxima utiliza o modelo neoclássico de crescimento para estabelecer a conexão entre a relação capital trabalho e a PTF.

\section{Relação Capital Trabalho e Produtividade Total dos Fatores}

A versão padrão do modelo básico de crescimento é caracterizada por um agente representativo que maximiza o valor presente de seu fluxo de utilidade esperada, a produção ocorre por meio de uma tecnologia que apresenta rendimentos decrescentes. Os agentes são expostos a um choque estocástico originado por uma distribuição conhecida por todos. Os mercados trabalham em

7 Como exemplo compare a repercussão da Política de Desenvolvimento Produtivo (PDP) com o Programa de Aceleração do Crescimento (PAC). Enquanto a primeira busca aumento da produtividade o segundo é claramente focado em investimento. 
concorrência perfeita e os preços se ajustam automaticamente ás condições de oferta e demanda.

Formalmente o problema da família representativa pode ser descrito como:

$$
\begin{aligned}
& \operatorname{\operatorname {ax}} E_{0}\left\{\sum_{t=0}^{\infty} \beta^{t} u\left(C_{t}, L_{t}\right)\right\} \\
& \text { s.a } C_{t}+X_{t}=A_{t} K_{t}^{\theta} L_{t}^{1-\theta}
\end{aligned}
$$

onde $C$ representa o consumo, $L$, as horas trabalhadas, $A$ a produtividade total dos fatores, $X$ o investimento e $K$ o estoque de capital. Os parâmetros são tais que $\beta$ representa o fator de desconto, e $\theta$ a participação da renda do capital na renda total. $\mathrm{O}$ estoque de capital evolui de acordo com:

$$
K_{t+1}=(1-\delta) K_{t}+X_{t}
$$

onde $\delta$ é a depreciação, a população cresce a uma taxa $n$.

Dividindo todas as variáveis por $A_{t} L_{t}$ e representado a razão pela letra minúscula correspondente á letra original o problema acima pode ser representado pela seguinte Equação de Bellman:

$$
V\left(k_{t}\right)=\max _{k_{t+1}, l_{t}}\left\{u\left(A_{t} k_{t}^{\theta} l_{t}^{1-\theta}+(1-\delta) k_{t}-(1+n \chi 11+\gamma) k_{t+1}\right)+\beta V\left(k_{t+1}\right)\right\}
$$

A condição de primeira ordem associada a $k_{t+1}$ determina uma Equação de Euler que pode ser escrita na forma:

$$
\frac{K_{t+1}}{L_{t+1}}=\left[\frac{\theta A_{t}}{\frac{(1+n)(1+\gamma)}{\beta} \frac{u_{t}}{u_{t+1}}-(1-\delta)}\right]^{\frac{1}{1-\theta}}
$$

Por meio da equação 8 observa-se que a dinâmica da relação capital trabalho depende da evolução da produtividade total dos fatores, da taxa de substituição intertemporal e de alguns parâmetros. Sendo assim, políticas que levem a um aumento da produtividade total dos fatores levam a um aumento da relação capital trabalho.

Considerando uma função de utilidade do tipo $u\left(C_{t}, L_{t}\right)=\frac{C^{1-\sigma}-1}{1-\sigma}+g(L)$ a taxa de substituição intertemporal será dada por:

$$
\frac{u_{d}}{u_{t+1}}=\left(\frac{C_{t+1}}{C_{t}}\right)^{\sigma}=\left(\gamma_{c}-1\right)^{\sigma}
$$

onde $\gamma_{d}$ representa a taxa de crescimento do consumo.

Pelas equações 8 e 9, quando a PTF fica estagnada, a única forma de aumentar a relação capital trabalho seria por meio de uma redução na taxa de crescimento do consumo. O Gráfico 2 mostra o comportamento da taxa de

8 Cole et alli (2005) trabalha com a mesma expressão para a relação capital trabalho, entretanto este autor considera que a PTF é da forma $A_{t}=\eta \mathrm{A}_{t}$, onde $\mathrm{A}_{t}$ é a PTF do país líder, no caso Estados Unidos, e $n$ é um número entre zero e um. 
crescimento do consumo no Brasil entre 1971 e 1980. Para evitar problemas com preços relativos utilizou-se a série de consumo a valores constantes de 1980, conforme disponibilizada pelo IPEA ${ }^{9}$, esta série cobre os período de 1970 a 2008, a figura encerra em 1980, pois este foi o ano que se encerrou a tendência acentuada de crescimento da relação capital trabalho.

Gráfico 2 - Taxa de Crescimento do Consumo, Brasil 1971 - 1980

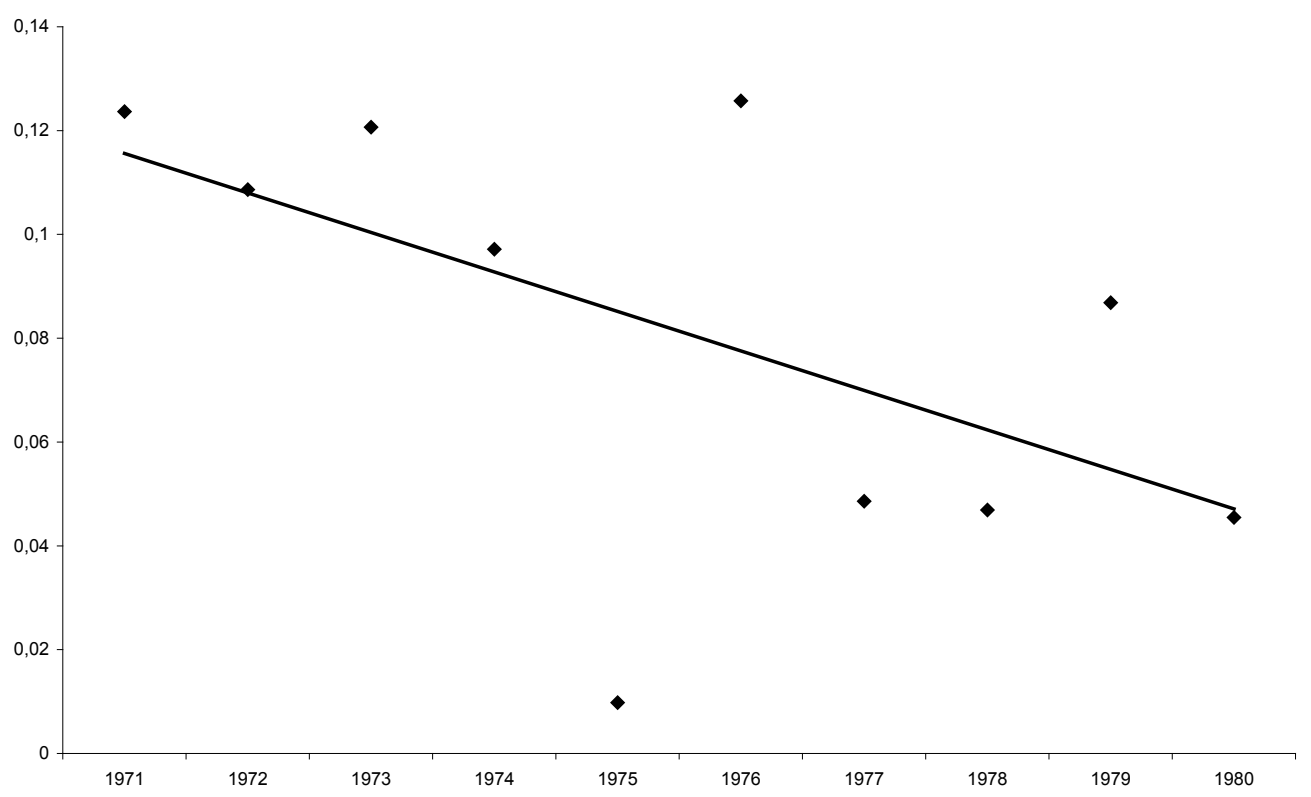

Fonte: Elaboração própria como base nos dados do Ipeadata e Contas Nacionais.

O Gráfico 2 sugere que, de acordo com o previsto pelo modelo neoclássico de crescimento, a elevação da relação capital produto se deu por meio de um sacrifício no consumo. Para reforçar este ponto vale notar que em 1970 o consumo representava 83\% do PIB, em 1980 este valor havia caído para 78\%.

A redução no consumo e na taxa de crescimento do consumo foi parcialmente induzida por políticas pública ${ }^{10} \mathrm{e}$ não o resultado de uma decisão ótima das famílias. É provável que as características políticas da época tenham inibido uma reação mais forte da sociedade a política de redução forçada do consumo. A viabilidade deste tipo de política em uma democracia permanece uma incógnita a ser estudada.

A discussão acima sugere que a elevação da relação capital trabalho pode ser obtida por meio da elevação da produtividade total dos fatores sem que seja necessário sacrificar consumo, e possivelmente, bem-estar. Ficou estabelecida, por meio do modelo neoclássico de crescimento econômico, a relação entre acumulação de capital e PTF. Na próxima seção um modelo semelhante ao descrito será utilizado para, por meio de simulações, avaliar o impacto da PTF no investimento.

9 www.ipeadata.gov.br

10 Um exemplo desta redução forçada do consumo foi a poupança forçada introduzida pela criação do FGTS pela Lei n. 5.107 de 13/09/1966. 


\section{Produtividade Total dos Fatores e Investimento}

O modelo desta seção é uma versão ligeiramente modificada do modelo anterior. Os indivíduos escolhem uma seqüência de consumo e de horas trabalhadas, $h$, de forma a resolver o problema:

$$
\begin{gathered}
\max E_{0}\left\{\sum_{t=0}^{\infty} \beta^{t}(1+n)^{t}\left[\ln \left(c_{t}\right)+\alpha \ln \left(1-h_{t}\right)\right]\right\} \\
\text { s.a } c_{t}+x_{t}=z_{t}(1+\lambda)^{(1-\theta) t} k_{t}^{\theta} h_{t}^{1-\theta}
\end{gathered}
$$

onde $z_{t+1}=1-\rho+\rho z_{t}+\varepsilon_{t}$, com $0<\rho<1$ e $\varepsilon_{t}$ é um ruído branco. A regra de movimento do capital é mesma da anterior.

Como o modelo não permite solução analítica será feita uma aproximação linear-quadrática para aproximar as funções políticas em uma proximidade do estado estacionário. A função política permite escrever a o investimento em função das variáveis de estado do modelo, capital e PTF, desta forma poderá ser utilizada para avaliar o impacto da PTF no investimento. A calibração seguirá Bugarin et alli (2007) ${ }^{11}$. A taxa de crescimento da população é de 2,39\% a.a., enquanto a taxa de crescimento da produtividade é de 1,3\% a.a. A participação da renda do capital na renda total é igual a 0,4 , a taxa de depreciação é de $9 \%$, o fator de desconto, calculado a partir da regra de movimento do capital, é 0,85 e $\alpha$ é igual a 1,57. Estes parâmetros garantem que a economia artificial reproduz e relação capital produto, cuja média para o período de 1950 a 2005 foi de 2,07, e a fração do dia dedicada ao trabalho observada na economia brasileira.

Gráfico 3 - Taxa de Investimento no Brasil, 1970 - 2005

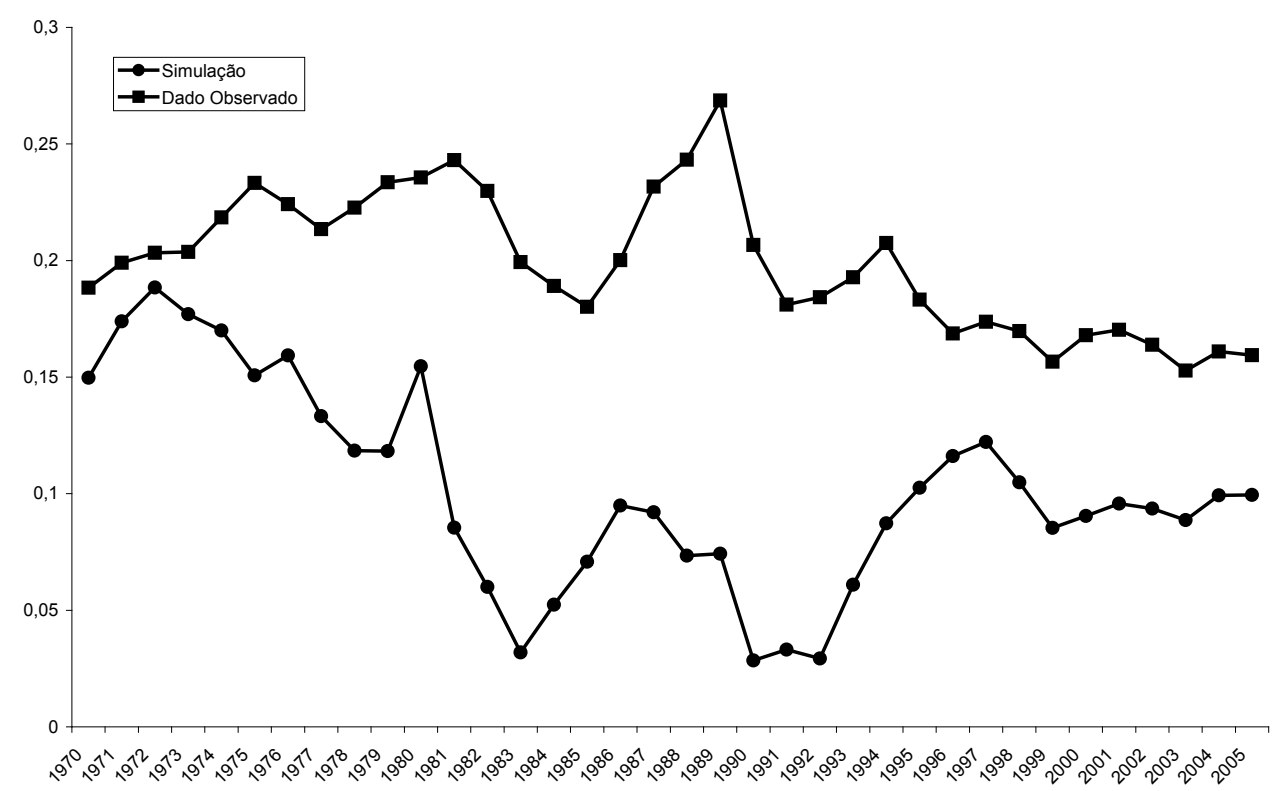

Fonte: Elaboração própria como base nos dados do Ipeadata e Contas Nacionais.

11 Os autores trabalham com o mesmo modelo utilizado nesta seção, de forma que não há inconsistência entre os valores do parâmetros. 
O Gráfico 3 mostra os valores observados para a taxa de investimento no Brasil bem como os obtidos por meio da simulação do modelo. Como esperado a taxa de investimento simulada é consistentemente mais baixa do que a taxa de investimento observada. Uma interpretação para este resultado é que a taxa de investimento ótima, induzida pela PTF, seria menor do que a observada.

Teoricamente um desempenho mais favorável da PTF poderia ter elevado as taxas de investimento sem a necessidade de recorrer a mecanismos de poupança forçada e sem sacrificar a taxa de crescimento do consumo. Para quantificar esta possibilidade são feitas simulações de dois cenários alternativos para a evolução da PTF. O primeiro supõe um aumento médio de 1\% na taxa de crescimento da PTF entre 1950 e 2005, o segundo considera um aumento médio de 4\% nesta taxa. O Gráfico 4 ilustra estes dois cenários, os valores observados e o caso básico.

Gráfico 4 - Cenários para Taxa de Investimento, 1970 - 2005

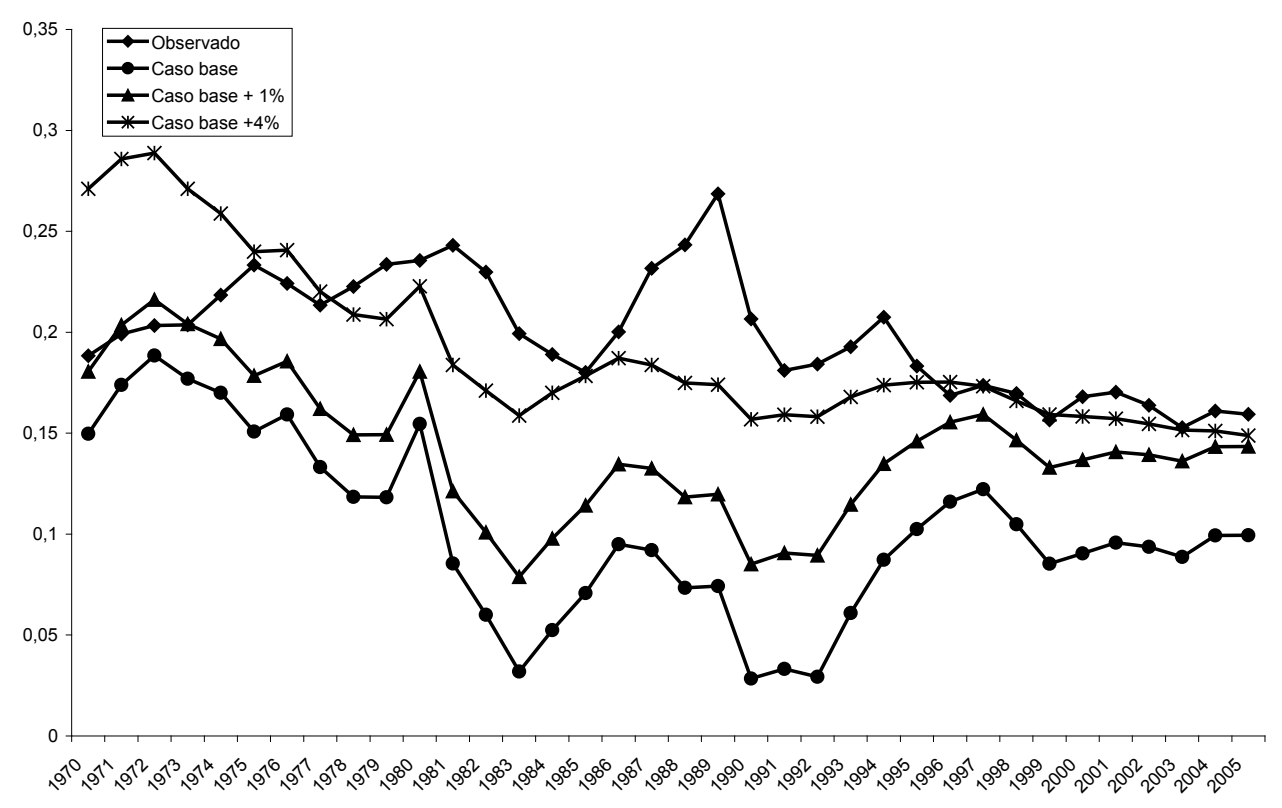

Fonte: Elaboração própria como base nos dados do Ipeadata e Contas Nacionais.

O caso mais próximo ao observado ocorre quando a PTF cresce a uma taxa $4 \%$ maior do que a observada, ou seja, a PTF cresce aproximadamente 4,7\% ao ano entre 1950 e 2005. Este caso deixaria a evolução da PTF brasileira semelhante á da coreana, que cresceu aproximadamente $4,6 \%$ ao ano entre 1953 e $2003^{12}$. Desta forma teria sido possível obter altas taxas de investimento sem as políticas de acumulação forçada de capital desde que a PTF no Brasil tivesse crescido de forma semelhante á de países como a Coréia.

A década de 1980 apresenta o maior desafio para o modelo neoclássico de crescimento em termos de reproduzir o comportamento da taxa de investimento no Brasil. Mesmo com a PTF seguindo padrões coreanos o modelo subestima a taxa de investimento neste período. Bugarin et alli (2007) argumentam que parte da dinâmica de acumulação de capital neste período pode ser explicada

12 Ver Ellery Jr. (2009). 
por mudanças nos preços relativos de bens de capital, particularmente quanto à construção civil. Feita a correção ocorre uma redução na taxa de investimento da década de 1980 e, especificamente, desaparece o pico de $1989^{13}$.

Outro ponto relevante que pode ser avaliado a partir de simulações do modelo neoclássico é o quanto um aumento da PTF afeta a trajetória do produto per-capita. Cole at alli (2007) argumentam que a maior restrição sobre o crescimento da América Latina advém das baixas taxas de crescimento da produtividade. Em particular os autores mostram que os efeitos da escassez de capital, físico ou humano, são pequenos quando comparados aos da PTF. Ellery Jr (2009) chega ao mesmo resultado em análise específica sobre o Brasil.

O Gráfico 5 mostra o comportamento do produto per-capita brasileiro em comparação ao que teria ocorrido se a PTF brasileira tivesse crescido a taxas semelhantes á dos Estados Unidos, que o caso onde se acrescenta 1\% na taxa de crescimento da PTF brasileira, porém sem as políticas de crescimento forçado. Note-se que, neste caso, a produto atual estaria próximo do observado, porém a trajetória teria apresentado menos picos e quedas abruptas.

Gráfico 5 - Produto per-capita no Brasil, 1950 - 2005

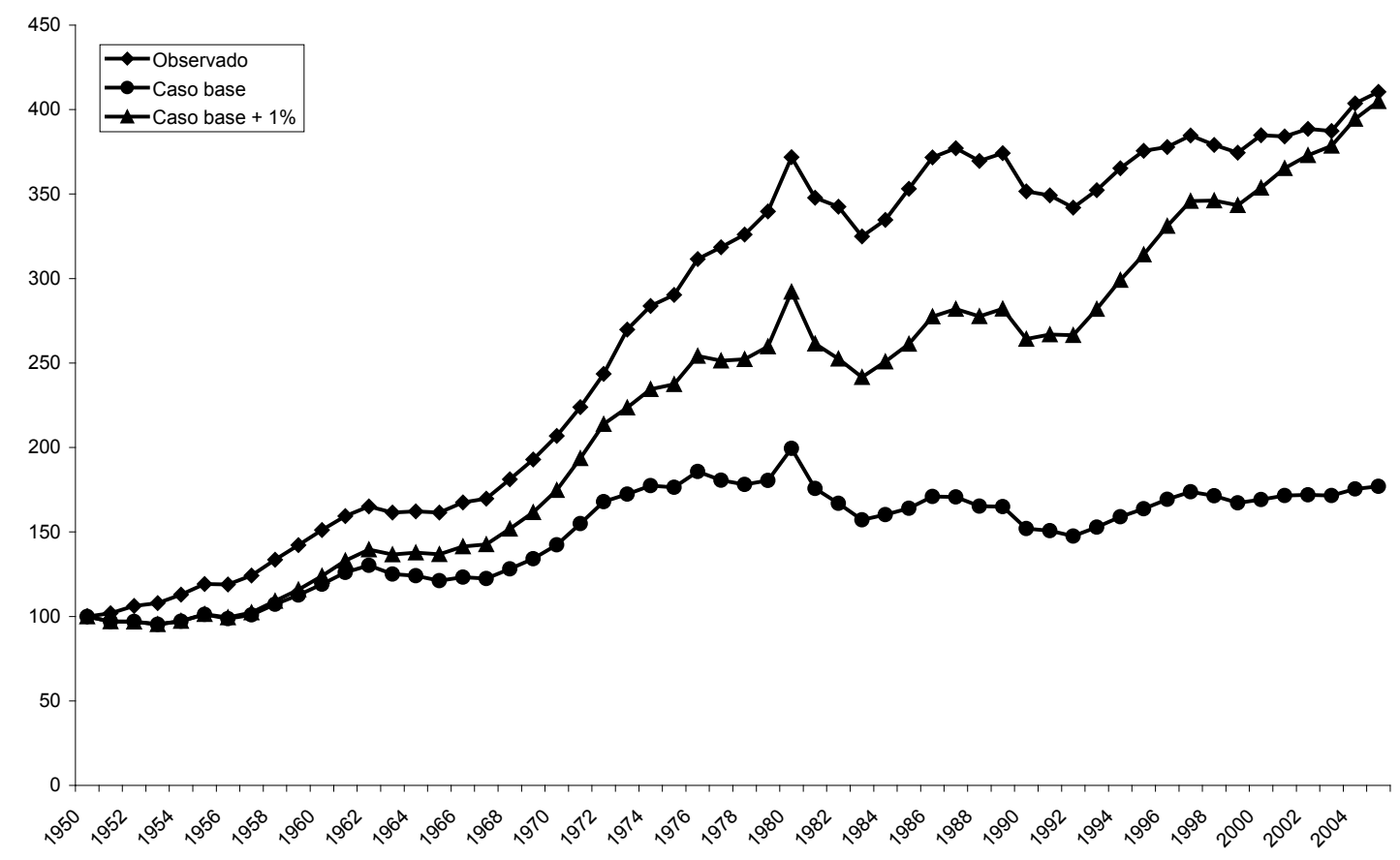

Fonte: Elaboração própria como base nos dados do Ipeadata e Contas Nacionais.

Caso o fraco desempenho da PTF tenha sido causado por conseqüência das políticas de crescimento forçado adotadas no pós-guerra, hipótese discutida em Cole et alli (2007), pode-se dizer que todo o ganho de crescimento do pósguerra foi perdido nas década perdidas de 1980 e 1990. O efeito negativo pode ser ainda mais grave se, na ausência destas políticas, a PTF no Brasil tivesse seguido trajetória semelhante á da Coréia. O Gráfico 6 ilustra este caso $^{14}$.

13 De fato a taxa de investimento em 1989 chega a a $27 \%$, mas cai para $19 \%$ se feita a correção pelos preços relativos. 14 Para facilitar a visualização a Figura 6 utiliza escala logarítmica. Para efeitos de comparação de valores absolutos, o último valor da série observada é de 410,5 contra 3894,4 da série simulada. 
Gráfico 6 - Produto per-capita no Brasil, 1950 - 2005

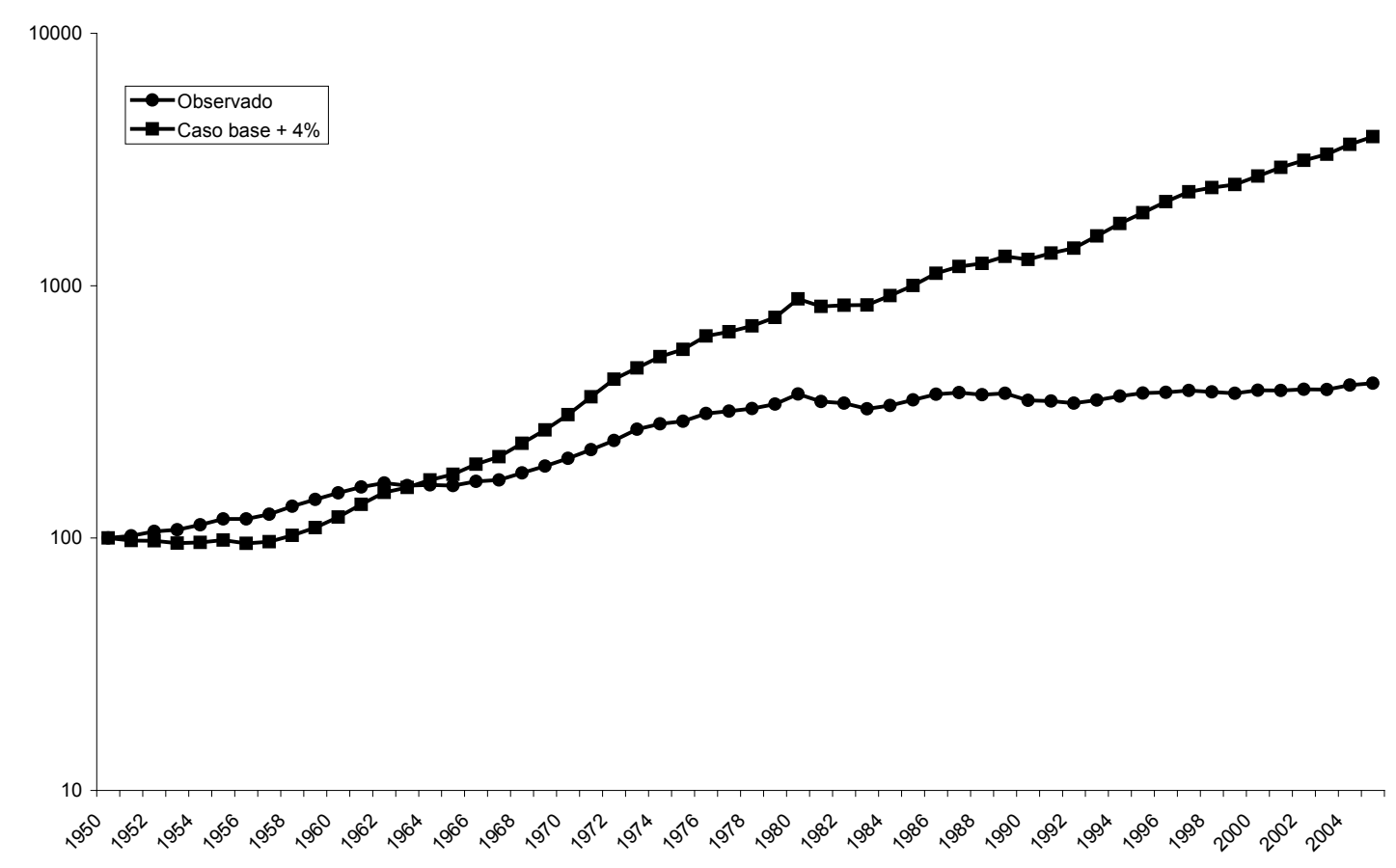

Fonte: Elaboração própria como base nos dados do Ipeadata e Contas Nacionais.

De acordo com as simulações ilustradas na Figura 6, se o Brasil tivesse implementado um conjunto de políticas capazes de fazer com que a PTF crescesse acima da dos Estados Unidos, especificamente com valores próximos aos observados na Coréia, o produto per-capita de 2005 seria da ordem de 9,5 vezes o observado naquele ano. Naturalmente nada garante que tal conjunto de políticas seria factível para o Brasil, entretanto o exercício ilustra a importância do aumento da taxa de crescimento da PTF para engendrar um processo de crescimento sustentável no Brasil.

\section{Conclusão}

Este trabalho avaliou o impacto da PTF na dinâmica de acumulação de capital e crescimento do produto per-capita no Brasil. Inicialmente, por meio de exercício de contabilidade do crescimento, mostrou-se que a acumulação de capital preponderou sobre a PTF na determinação do crescimento do PIB percapita. Esta característica, comum a outros países da América Latina, estaria associada a uma falta de sustentabilidade das taxas de crescimento, o que de fato aconteceu.

$\mathrm{Na}$ terceira seção foram exploradas as relações teóricas entre a razão capital trabalho e a PTF. Nesta seção ficou estabelecia a relação entre estoque de capital e PTF, em particular, foi visto que quanto maio a PTF maior será a razão entre capital e trabalho de equilíbrio. Dados da economia brasileira mostram uma elevação da relação entre capital e trabalho, porém não mostram 
um aumento da PTF compatível com esta elevação. As condições de equilíbrio do modelo neoclássico implicam que esta combinação é possível desde que ocorra uma redução na taxa de crescimento do consumo, esta redução é observada na economia brasileira durante o período em questão. O resultado que o modelo neoclássico subestima o investimento no Brasil está presente em Bugarin et ali( 2007), aqui foi mostrado que o aumento do estoque de capital foi de fato obtido por meio da redução na taxa de crescimento do consumo.

A quarta seção tratou da relação entre PTF e investimento e produto per-capita. Para isto foram utilizadas simulações do modelo neoclássico de crescimento econômico. Inicialmente constatou-se que a taxa de investimento no Brasil ficou muito acima da prevista pelo modelo básico, que seria a taxa induzida pela PTF. O resultado se mantém mesmo quando é feita a hipótese que a taxa de crescimento da PTF aumenta em 1\% e 4\%, tornando-a próxima da observada nos Estados Unidos e na Coréia, respectivamente. Quando da análise do produto per-capita foi visto que, mesmo com a menor taxa de investimento, o produto teria crescido de forma semelhante à observada no caso da PTF ter crescido a uma taxa 1\% maior e seria quase dez vezes maior que o observado se a PTF tivesse crescido a uma taxa 4\% maior do que a que ocorreu.

A conclusão geral é que políticas ou mudanças institucionais que induzam a um aumento da PTF têm um maior potencial de afetar o crescimento do que políticas focadas na taxa de investimento. Esta conclusão está de acordo com a encontrada em Cole et alli (2007) e Ellery Jr e Teixeira (2013). Entretanto nada pode ser dito sobre a natureza das políticas que possam induzir o crescimento da PTF, aceitas as conclusões deste trabalho, determinar os efeitos das políticas públicas sobre a PTF é, sem dúvida, o desafio central para os estudiosos do crescimento econômico do Brasil.

\section{Referências}

Bugarin, M., Ellery Jr., R. G., Gomes, V., \& Teixeira, A. (2007). The Brazilian depression in the 1980s and 1990s. Em Great Depressions of the Twentieth Century. Timothy Kehoe e Edward Prescott (org.). Federal Reserve Bank of Minneapolis, Minnesota.

Bugarin, M., Ellery Jr., R. G., \& Gomes, V. (2005). Long-run implications of Brazilian capital stock and income estimations. Brazilian Review of Econometrics, v.25 (1).

Cole, H. L., Ohanian, L. E., Riascos, A., \& Schmitz, J. (2005). Latin America in the rearview mirror. Journal of Monetary Economics, v.52 (1).

Cooley, T. \& Prescott, E. (1995). Economic growth and business cycle. Em Frontiers of Business Cycle Research. Thomas Cooley (org.). Princeton University Press, Princeton.

Ellery Jr., R. G. (2009). Estratégias para o Crescimento da Economia Brasileira. Em Crescimento Econômico: Setor Externo e Inflação. Renaut Michel e Leonardo Carvalho (org.). Instituto de Pesquisa Econômica Aplicada, Brasília. 
Ellery Jr., R. G \& Teixeira, A. (2013). O Milagre, a Estagnação e a Retomada do Crescimento: As Lições da Economia Brasileira nas Últimas Décadas. Em Desenvolvimento Econômico: Uma Perspectiva Brasileira. Fernando Veloso, Pedro Ferreira, Fabio Giambiagi e Samuel Pessoa (org). Elsevier, Rio de Janeiro.

Ellery Jr., R. G., Gomes, V. \& Sachsida, A. (2002). Business cycles fluctuations in Brazil. Revista Brasileira de Economia, v.56 (2).

Gollin, D. (2002) Getting income shares right. Journal of Political Ecoonomy, v110 (2), p. 458-74.

Gomes, V., Pessôa, S., \& Veloso, F. (2003). Evolução da produtividade total dos fatores na economia brasileira: uma análise comparativa. Pesquisa e Planejamento Econômico, v.33 (3).

Kehoe, T. \& Prescott, E. (2007). Great Depressions of the Twentieth Century. Federal Reserve Bank of Minneapolis, Minnesota.

Morandi, L. \& Reis, E. (2004). Estoque de capital fixo no Brasil - 1950 - 2002. Em Anais do XXXII Encontro Nacional de Economia, João Pessoa. Anpec.

Pessôa, Samuel, Pessoa, Silvia, \& Rob, Rafael. (2003) Elasticity of substitution between capital and labor: a panel data approach. Ensaios Econômicos n.494, EPGE/FGV-RJ.

Solow, R. (1956). A contribution to the theory of economic growth. Quarterly Journal of Economics, v.70 (1).

Solow, R. (1957). Technical change and aggregate production function. The Review of Economics and Statistics, v.39 (3). 1957.

Young, A. (1995). The tyranny of numbers: confronting the statistical realities of the East Asian growth experience. Quarterly Journal of Economics, v110 (august), p. 641-80. 
